\title{
EDITORIAL MOVILIDADES, ETNICIDADES, Y FRONTERAS NACIONALES EN EL SIGLO XXI. FAMILIAS CHIPAYA EN EL NORTE CHILENO
}

\author{
Vivian Gavilán Vega ${ }^{1}$
}

...que verdaderamente somos urus y según su etimología somos gente de agua, que Dios y la naturaleza nos designó el corto recinto de Ayparavi y la laguna donde vivimos y con su producto nos alimentamos.

Manuel Perca (1825) en Wachtel (2001)

Las nuevas oleadas de migrantes a Chile provenientes de los países vecinos y en los últimos años de Centroamérica es hoy una preocupación para el Estado. La población inmigrante ha comenzado a ser centro de atención para el diseño de políticas públicas regionales. Además, el fenómeno migratorio en el nuevo escenario internacional ha sido estudiado por las ciencias sociales desde hace ya varias décadas, siendo hoy un tópico de investigación importante. Sin duda, constituye un problema social que merece ser abordado en sus diferentes aristas.

Los desplazamientos poblacionales en el sur andino son de larga data. Movimientos de grupos que se trasladan a lo largo de la costa y de esta hacia la cordillera de los Andes y Altiplano, y viceversa, han sido documentados por historiadores y arqueólogos. Se trata de estrategias familiares y comunales que se relacionan con la búsqueda de mejores condiciones de vida. Lo novedoso es que en cada contexto histórico adquieren especificidades que vale la pena conocer.

La imposición de las fronteras de los Estadosnaciones modernos constituye uno de los últimos límites territoriales en los que se mueven los pueblos aimara, quechua y chipaya en la franja territorial que comprende Perú, Bolivia y Chile. En un contexto en el que los Estados-naciones son la principal forma de organización social, económica, política y cultural, la cuestión de la convivencia de las nacionalidades boliviana, peruana y chilena en un mismo territorio adquiere relevancia, pues los procesos identitarios asociados a la nación, se superponen a las relaciones interétnicas preexistentes.

La profundización del capitalismo en la región ha favorecido la flexibilidad de las fronteras y las tecnologías de comunicación han facilitado los vínculos entre los lugares de origen y de destino, promoviendo así una mayor población movilizada y con voluntad para asentarse de manera permanente en las ciudades de acogida. La presencia de grupos de personas provenientes de Perú, Bolivia, Colombia y recientemente de República Dominicana es cada vez más visible, transformando el paisaje cultural de las ciudades nortinas.

En este panorama, el énfasis puesto en las nacionalidades tiende a eclipsar las identidades étnicas y/o de género. Las identidades étnicas, esto es, aquellas que se han construido históricamente y que se asocian a los pueblos originarios, suelen ser invisibles en los análisis de las migraciones que realizan los diseñadores de políticas públicas. Me refiero a las colectividades que descienden o dicen descender de las poblaciones indígenas del sur andino y que ocuparon los territorios hoy administrados por Chile, Bolivia y Perú. El diseño de políticas inclusivas y con enfoque de derechos debiera considerar estas historias. No es lo mismo el fenómeno migratorio de la población de Ecuador o Colombia hacia Chile, que el de las zonas transfronterizas, como es el caso del sur del Perú y/o de la zona centro oeste de Bolivia.

Uno de los ejemplos paradigmáticos en el que se refleja la importancia de la historia local de los desplazamientos transfronterizos es el de las familias de origen chipaya. Se trata de una colectividad cuyo pueblo central se ubica en la zona altiplánica

1 Departamento de Antropología, Universidad de Tarapacá, 18 de Septiembre 2222, Casilla 6-D, Arica - Chile. viviangav@yahoo.com 
de la actual Bolivia. Como veremos más adelante, registran, desde tiempos coloniales, movimientos migratorios por una amplia zona que considera el altiplano y las vertientes oriental y occidental de los Andes.

Una aproximación a la situación de la población de origen chipaya en Chile permite sugerir la hipótesis de que la particularidad que adquiere la inmigración de este grupo en la actualidad es la tendencia a la permanencia definitiva en las diferentes ciudades y pueblos del norte grande ${ }^{1}$. Ello no implica la desvinculación de la comunidad de origen. Aunque ya en la primera mitad del siglo $\mathrm{XX}$ varios investigadores auguraban la desaparición de este pueblo por efecto de su movilidad en busca de trabajo y ello no se produjo, hoy se observa que las nuevas generaciones han aumentado su nivel de escolaridad y sus expectativas de vida consideran un mayor bienestar material. La pregunta que cabe es si la comunidad chipaya se translocalizará hacia las zonas urbanas, como sus vecinos aimaras de Chile (cf. Carrasco y González 2014; González y Gundermann 2009; González et al. 2014; Gundermann y González 2009).

El Estado chileno considera solo su nacionalidad, la sociedad regional no distingue al interior de la población indígena ${ }^{2}$, las familias aimara chilenas los declara extranjeros y como descendientes de otro grupo cultural, pero del mismo pasado indígena. En tanto que los aimara de nacionalidad boliviana los consideran como connacionales, pero de un grupo cultural diferente. Los aimara, en su conjunto, los clasifican como chullpas, es decir, como colectividad que persiste en las formas de vida de los antepasados indios. En síntesis, otra clase de indios.

En el contexto de la desigualdad social estructural, los chipaya en Chile son vulnerables por su condición étnica, por su nacionalidad y por su estatus inmigratorio; especialmente las personas que no cuentan con residencia permanente, pues se desempeñan como trabajadores indocumentados. A pesar de esto, tanto las prácticas desplegadas por las familias chipaya para integrarse a la sociedad en Chile como las reflexiones que elaboran como ciudadanos y como indígenas expresan un manejo consciente de las reglas del juego que imponen los Estados-naciones. Las conversaciones sostenidas con mujeres y hombres chipaya residentes en la ciudad de Alto Hospicio y en las localidades de La Tirana y de Pintados muestran a personas con capacidad para sortear obstáculos y para buscar soluciones a sus problemas inmediatos. A pesar de la discriminación negativa que experimentan tanto por la sociedad nacional chilena como por los aimara, se desenvuelven entre relaciones interétnicas e internacionales. De aquí que no se puede desconocer la historia de los pueblos clasificados en tiempos coloniales como urus, en general, y la de los urus-chipaya, en particular. Se trata de un pueblo con una férrea voluntad por mantener su lengua y sus tradiciones $\mathrm{y}$, al mismo tiempo, con capacidades para enfrentar los contextos administrativos neocoloniales.

\section{Los Chipaya}

Los chipaya constituyen un grupo étnico ubicado en el salar de Coipasa. Administrativamente se ubican en el cantón Chipaya, provincia de Atahuallpa, departamento de Oruro, a 220 kilómetros al sureste de Oruro, Bolivia. El censo 2001 de Bolivia informa que el cantón de Chipaya registró una población de 1.814 habitantes, de los cuales 943 son hombres y 841 son mujeres; integrado por 855 viviendas. De acuerdo con esta fuente se compone de seis organizaciones comunitarias: Aranzaya, Ayllu Manasaya, Chipaya, Ayllu Unión Barra, Ayparavi y Vestrullani, las que incluyen las localidades de Aranzaya, Manasaya, Chipaya, Antiguo Ayparavi (Vito Pampa), Ayparavi, Estancia Vestrullani (CEPAL 2005).

Wachtel (1997) afirma que sería el último testimonio de la etnia Uru. Su sistema económico asocia esencialmente la cría de animales con la agricultura de quinua. La pesca y la caza juegan ahora un rol secundario. Agrega que por oposición a sus vecinos aimaras, los chipayas se identifican a sí mismos como jas-shoni, es decir, gente de agua, en oposición a los "hombres secos", quienes los rodean. Hasta la primera mitad del siglo XX tuvieron una relación conflictiva con las comunidades aimara, especialmente de Huachacalla; de quienes se habrían emancipado de la situación de servidumbre que mantenían desde la Colonia (Wachtel 2001).

Wachtel señala que la condición de uru explica en gran parte el tipo de relaciones interétnicas que establecen con sus vecinos aimara. Estos los consideran chullpa-puchu o "desechos" de la era presolar y excluidos de la humanidad actual (Watchel 1997:12). El autor plantea que el mito andino de los chullpas sugiere que son descendientes directos 
de una pre-humanidad, de una edad anterior a la de los hombres:

Este pueblo de chullpas, seres que, de acuerdo al mito original, poblaban la tierra antes de la aparición del sol. Cuando este surgió, todos los chullpas fueron quemados por el fuego celeste, salvo algunos de ellos que se refugiaron en los lagos. De estos sobrevivientes del primer juicio descienden los chipayas, así como los otros urus, últimos testigos en este mundo de una humanidad primordial (Watchel 1997:12).

El mito de origen de este pueblo va de la mano de los etnógrafos de inicios del siglo XX, quienes los describieron "primitivos" e "incivilizados". En 1936 Metraux señala:

Vive en Chipaya una raza primitiva de gentes que casi no mantiene contacto alguno con otros pueblos del altiplano. Sus vestidos, que pueden remontarse al siglo $\mathrm{XV}$, siguen siendo tan idénticos como los que cubren sus chullpas (Metraux citado en Pauwels 1998:60).

Así, las formas de vida de este pueblo nos permitían conocer el pasado de los aimara.

Los informes coloniales indican que los urus ocuparon gran parte del altiplano y otros lugares, pero que fueron cediendo terreno y desapareciendo, a causa principalmente del acoso de otros grupos como los aimaras y quechuas que se apoderaron de sus recursos naturales. Es probable que este acoso hacia los urus sea anterior al arribo de los españoles, y continuado durante la Colonia y República. Sabemos hoy que la autodesignación como indios urus así como nombrar su lengua como puquina, responde a las clasificaciones externas para efectos de administración colonial (Cerrón-Palomino 2006; Martínez 2011).

Cerrón-Palomino afirma que se definen a sí mismos como qhwasz-zh zhoñi o "hombres del agua", por oposición a los "hombres secos" o foráneos en general, a quienes se les conoce como tozha (Cerrón-Palomino 2006:18).

Los desplazamientos que realizan los habitantes de la colectividad chipaya, desde Santa Ana hacia la zona occidental de los Andes, han estado presentes a lo largo del siglo XX y continúan activos hoy, pero adquieren características particulares que sería importante investigar no solo para poner en juego las teorías de la etnicidad, de la nacionalidad y de la ciudadanía, sino también para comprender de mejor modo las prácticas de los pueblos originarios y los efectos que tienen las políticas económicas y sociales de los Estados-naciones en la vida de los mismos.

\section{La Movilidad de los Chipaya hacia el Norte de Chile}

La mayor parte de quienes han descrito la situación de este pueblo se refieren a los procesos migratorios hacia los valles occidentales, pampa salitrera o ciudades de la costa del norte chileno (Acosta 2001; Barrientos 2012; Cereceda 2010; Cerrón-Palomino 2006; Metraux en Pauwels 1998; Muñoz 2011; Wachtel 1997, 2001; Uhle 1922)³.

En 1894 Max Uhle visitó la zona y describe a los urus, nombre asignado en tiempos coloniales a los habitantes de las islas y que se ubicaban "en la costa pacífica entre Arica y Tarapacá, al sur del lago en Pucarani donde hablaban su idioma". Al momento de su exploración, identifica una "población de unos 500 uros en Chipaya al norte del salar de Coipasa, siendo este su centro más importante". Aclara que las áreas ocupadas por uros y puquinas eran bastante diferentes, lo mismo que sus lenguas (Uhle 1922:8).

Pauwels cita a Posnanky (1937) para señalar que de no haberse presentado la crisis del salitre, los chipayas hubieran emigrado definitivamente, dejando de ser chipayas (Pauwels 1998:56). CerrónPalomino (2006) argumenta que la precariedad de su economía obliga a los chipaya a buscar fuentes de ocupación fuera de su comunidad y que los varones se movilizan temporalmente hacia las valles precordilleranos de Chile. Cereceda (2010) señala también que muchos chipaya se aventuran hacia Chile a realizar trabajos temporales en las chacras de Chiapa, Camiña y otros valles y, por lo menos, hace unos 30 años todavía había enclaves chipaya en el ayllu Isluga, hoy territorio chileno. Agrega que a partir de la historia oral se puede sostener que los chipaya parecen haber dominado en el pasado un paisaje mucho más amplio que el que hoy habitan, que se extendía, posiblemente, hasta los bordes mismos del océano Pacífico.

Barrientos (1990:85-86) por su parte afirma que antes de la apertura de la educación secundaria en el poblado Chipaya, muchos jóvenes se trasladaron a Chile a cursar estudios secundarios. 
Un sacerdote franciscano, hoy párroco de la región de Tarapacá, norte chileno y que convivió con los chipaya sostiene que el principal motivo para el desplazamiento es tener oportunidades de trabajo. Un joven chipaya trabajador de las parcelas de Pintados en esta misma región declara que son los jóvenes los que más vienen a trabajar a Chile por temporadas, muchos de los cuales desean quedarse permanentemente.

Muñoz (2011) indica que el paso hacia Chile de los chipaya son las localidades aimara fronterizas (Isluga y Cariquima) y que la permanencia puede ser temporal, lo que corresponde a quienes están por períodos breves de trabajo en la agricultura de temporada; o puede ser definitiva. Quienes solicitan los permisos legales los hacen en el paso fronterizo de Colchane como turistas con el propósito de obtener el permiso de residencia.

Entre 2005 y 2008 y en el contexto de un estudio sobre identidades étnicas en la región entrevistamos a mujeres y a hombres chipaya -jóvenes y mayores- residentes en la provincia del Tamarugal. Argumentaban que salen de su comunidad por problemas económicos y en búsqueda de mejores condiciones de vida para sus hijos y por ello deciden venir a Chile.

Actualmente se desconoce cuántas personas de este pueblo residen en territorio chileno. Los entrevistados afirmaron que a lo menos la mitad de la población chipaya se encontraba dispersa en los valles agrícolas precordilleranos, los pueblos de Pintados y La Tirana, y en las ciudades de Iquique, Alto Hospicio o Antofagasta. Uno de los adultos señalaba:

...los inmigrantes han aumentado aquí en Chile, somos muchos, somos muchos chipayas... en Antofagasta son como una comunidad... y acá en Hospicio también, y en La Tirana también somos varios más... (T.F.).

Reconocen que varias generaciones acostumbraban trasladarse hacia la costa, sin embargo en los últimos años habría aumentado la cantidad de personas que deciden instalarse en el norte chileno. Uno de ellos nos cuenta que:

....antes nuestros abuelos siempre venían a hacer canje con la mercadería, con lana, a Chiapa, a veces creo que iban también a Calama también venían... además del terreno donde nosotros estamos en chipaya estamos muy descuidados... la gente crece más y los terrenos no aumentan, y nosotros estamos muy despegados de la superficie aimara... la gente multiplica, pero el terreno no multiplica, entonces muchas parejas vienen a Chile, por que allá van a querer tener su ganadito, su terreno, pero allá no hay vida, entonces se vienen para acá, y trabajan como nosotros.

Desde mi abuelo, desde mi bisabuelo como unos tres no más venían, pero desde mi abuelo ya venían un poquito más... un poquito más... ya cuando era mi papá ya casi todos venían... ya cuando mi papá era joven, tenia como 17 o 18 años, cuando era soltero, era como una moda así venir a Chile, ya traer algo a la casa... entonces ya de ahí empezaron, como que vieron que ya ganaba platita todo eso, empezaron a venirse pa acá... entonces ya empezaron a arreglarse sus documentos ya... ahora a edad mía ya casi todos tienen sus documentos ya, los que están acá, todos, no he escuchado ninguna persona que son bolivianos ahí, casi todos, no todos tampoco, así... yo creo que están haciendo recién también, hay amnistía, por ejemplo ahora salió amnistía, y ahí se han metido hartas personas igual... entonces yo creo que va a haber después, y van a seguir entrando personas, por que como es cerca entonces fácil vienen (E.M.).

El entrevistado se refiere a los diferentes estatus migratorios de sus compatriotas.

Una parte ha regularizado su residencia ante las autoridades chilenas, en tanto que otra permanece indocumentada; algunos pueden resolver su situación en períodos de amnistía declarados por el gobierno central de Chile y obtener la residencia definitiva.

Las mujeres casadas con hombres aimara de nacionalidad chilena, así como quienes consiguen la residencia por contratos de trabajo, se esfuerzan por conseguir un empleo independiente. Por lo general arriendan o compran locales de venta de verduras y otros en las ferias agropecuarias. Quienes no tienen permiso de residencia se cuidan de la pesquisa de la policía de migraciones y suelen ser peones de familias aimara o no indígena.

El proyecto de vida en Chile se asocia con mejoras en las condiciones de vida, lo que se expresa en aspiraciones para la vivienda propia, estudios 
o un buen trabajo. Aseguran que la educación es más adelantada que en Bolivia, especialmente por el manejo de computadores.

Los efectos de la indocumentación implica cárcel, deportación y explotación laboral. Por tanto las redes familiares y de amistades son importantes para la inserción en el espacio rural y urbano. El cambio de nombre parece constituir una estrategia para la permanencia en territorio chileno. Suelen ponerse nombres aimara, lo cual se ha convenido con sus empleadores.

Los entrevistados se sienten extranjeros en Chile. Tienden a agruparse como colectividad boliviana y entre sus actividades organizativas se halla la conformación de clubes deportivos de mujeres y hombres auspiciados por los municipios locales. Por medio del deporte establecen relaciones con las autoridades gubernamentales para conseguir beneficios sociales, especialmente canchas de fútbol y vivienda. De este modo, los clubes deportivos constituyen una plataforma para la sociabilidad. E.M. cuenta que llegaron a conformar nueve equipos de fútbol, buscaron canchas y solicitaron apoyo a los vecinos y a los Alcaldes:

...en la cancha que está para el lado de La Negra... ahí tienen su cancha, han entrado en acuerdo con los chilenos, y les han dejado un pedacito de espacio para que jueguen, en cancha, ahí juegan. ... porque el año pasado tenía nueve capitanes, ... entonces hemos solicitado a unos conocidos chilenos, entonces dijeron ¿ustedes ya tienen sus documentos chilenos ya?, entonces le dijimos al alcalde de Pozo Almonte nosotros queremos jugar, queremos compartir, en partido, con los jóvenes ahí, nosotros somos de La Tirana... entonces podemos decir que cumple la autoridad, nosotros somos de Bolivia pero tenemos aquí nuestros documentos ya, queremos compartir en partido, entonces así dio vestimenta, para los hombres y las mujeres (E.M.).

Las formas que adquieren las competencias de fútbol son particulares:

...el año pasado... el equipo de La Tirana tiene su propio uniforme de color verde, porque $\mathrm{La}$ Tirana es verde por el monte, entonces tenemos el color verde, y tenemos el color amarillo para las mujeres, también tenemos nuestra bandera, el año pasado hicimos campeonato y tuvimos de premio en vez de una copa tuvimos una olla, entonces ese fue el premio, una olla grande (E.M.).

La experiencia como inmigrantes los lleva a reflexionar acerca de la situación política actual tanto de Bolivia como de Chile de tal forma que les oriente a la búsqueda de estrategias para mejorar su posición social. Por un lado hablan de las dificultades que tiene Bolivia para resolver los problemas sociales. Piensan que la gran cantidad de personas campesinas residentes en las ciudades y en el campo condujo a elegir un presidente indígena; quien por su condición defiende los derechos indígenas. Refiriéndose al presidente Evo Morales postulan que la condición indígena en Bolivia implica derechos sociales y culturales que deben respetarse. En este sentido, dejar de ser chipaya y solo ser boliviano implicaría perder los posibles beneficios sociales que reciben por ser indígenas.

Un dirigente plantea la posibilidad de luchar por el reconocimiento como agrupación étnica en Chile. Se pregunta: ¿Qué nos conviene más ser chipaya o bolivianos no más?

Como extranjeros residentes, desde hace años debaten las ventajas que podría tener reconocerse como pueblo originario en Chile. La historia local de los chipaya se ha elaborado principalmente desde el exterior. Tanto los discursos etnificadores como las prácticas de exclusión socioeconómica, sociopolítica y sociocultural provienen del plan colonialista de los Estados-naciones y de las sociedades regionales. No se sabe mucho de las experiencias vividas por las familias a lo largo del siglo XX. Son escasos los trabajos que informen respecto de los proyectos de vida de los actuales chipaya. Desde el punto de vista de la ciudadanía, de las etnicidades y de los derechos de los pueblos originarios vale la pena reflexionar sobre la actual frontera impuesta por los Estados bolivianos y chilenos.

Si consideramos la diversidad de autores que han documentado la movilidad de las familias chipaya hacia el Pacífico, vemos que el apego territorial no emerge solo como un pasado nostálgico, sino también considera prácticas concretas de apropiación de una vasta zona del sur andino (del altiplano, los valles, la pampa del tamarugal y las ciudades de la costa) para su reproducción social, económica y cultural. Aun cuando vuelven a su localidad de origen, en un ir y venir, pareciera que la aspiración a vivienda 
en las ciudades nortinas nos habla de un aspecto particular de las migraciones en el siglo XXI. El manejo que tienen de los subsidios habitacionales estatales y otras opciones de compra, dan fe de ello (cf. Muñoz 2011).

Por lo demás, el caso chipaya nos pone ante una realidad escasamente estudiada; esto es, la relación de las mayorías y minorías étnicas en el contexto del Estado Plurinacional. Lara Barrientos (2012) acierta al plantear que es importante considerar la existencia de realidades indígenas distintas con relación a unos y otros. En este camino, afirma, se debe pensar en políticas diferenciadas para las minorías, que más allá del volumen de población, representan el fundamento de aquello que llamamos diversidad. El autor insiste en que la lucha contra el racismo y toda forma de discriminación incluye los prejuicios y discriminación que históricamente han servido a los pueblos para dominarlos, someterlos o excluirlos. No solo se trata, entonces, de las vinculaciones que el pueblo chipaya establece con el Estado boliviano, sino también con el Estado chileno. No solo se relaciona con los Estadosnaciones, sino también con las mayorías étnicas con las cuales se ha relacionado desde hace más de cuatro siglos.

\section{Referencias Citadas}

Acosta, O. 2001. La muerte en el contexto uru: el caso Chipaya. Chungara Revista de Antropología 33:259-270.

Barrientos, I. 1990. Chipaya Reliquia Viviente. Estudio Monográfico Chipaya. Editora Quelco, Oruro.

Carrasco, A. M. y H. González 2014. Movilidad poblacional y procesos de articulación rural-urbano entre los aymara del norte de Chile. Si Somos Americanos. Revista de Estudios Transfronterizos 2:217-231.

CEPAL 2005. Documentos de proyectos Los pueblos indígenas de Bolivia: diagnóstico sociodemográfico a partir del censo. Santiago de Chile. http://repositorio.cepal.org/bitstream/ handle/11362/3566/S2009029_es.pdf?sequence=1

Cereceda, V. 2010. Una extensión entre el altiplano y el mar. Relatos míticos chipaya y el norte de Chile. Estudios Atacameños 40:101-130.

Cerrón-Palomino, R. 2006. El Chipaya o la Lengua de los Hombres de Agua. Fondo Editorial Pontificia Universidad Católica del Perú, Lima.

Dedenbach-Salazar Sáenz, S. 2007. The Andean Uru-Chipaya Language (State of Research and Bibliography 2007). http:// dobes.mpi.nl/project_data/chipaya/Chipaya $\% 20$ state $\% 20$ of $\% 20$ research $\% 20$ and $\% 20$ bibliography $\% 20 \% 28$ Dedenbach, $\% 20$ 19-05-07\%29.pdf

González, H. y H. Gundermann 2009. Acceso a la propiedad de la tierra, comunidad e identidades colectivas entre los aymaras del norte de Chile (1821-1930). Chungara Revista de Antropología Chilena 41:51-70.
González, H., H. Gundermann y J. Hidalgo 2014. Comunidad indígena y construcción histórica del espacio entre los Aymara del Norte de Chile. Chungara Revista de Antropología Chilena 46:233-246.

Gundermann, H. y H. González 2009. Sociedades indígenas y conocimiento antropológico: aymaras y atacameños de los siglos XIX y XX. Chungara Revista de Antropología Chilena 41:113-164.

Lara Barrientos, M. 2012. Discriminación hacia minorías étnicas: el caso de los urus del lago Poopó. Tinkazos 15:57-78. Recuperado en 27 de marzo de 2016, de http://www.scielo.org.bo/scielo. php?script=sci_arttext\&pid=S1990-74512012000100004\&ln $\mathrm{g}=\mathrm{es} \& \mathrm{t} \operatorname{lng}=\mathrm{es}$.

Martínez, J.L. 2011. Gente de la Tierra de Guerra. Los Lipes en las Tradiciones Andinas y el Imaginario Colonial. DIBAM y PUC, Lima.

Muñoz, C. 2011. Los urus-chipaya en Chile. Revista de Ciencias Sociales 27:7-20.

Pauwels, G. 1998. Los últimos chullpas. A. Métraux en Chipaya (enero-febrero de 1931). Eco Andino 6:41-82.

Wachtel, N. 1997. Dioses y Vampiros. Regreso a Chipaya. Fondo de Cultura Económica, Ciudad de México.

Wachtel, N. 2001. El Regreso de los Antepasados. Los Indios Urus de Bolivia, del Siglo XX al XVI. Ensayo de Historia Regresiva. Fondo de Cultura Económica, Ciudad de México.

Uhle, M. 1922. Fundamentos étnicos de Arica y Tacna. Imprenta Banco Central, Quito.

\section{Notas}

1 Los datos fueron recolectados en el contexto del proyecto Fondecyt No 1050143 entre los años 2005 y 2008.

2 En las últimas décadas las políticas estatales de reconocimiento de los pueblos originarios y las prácticas de las organizaciones que reivindican derechos étnicos han promovido a la comunidad aimara, quechua y afrodescendiente.
3 Un excelente trabajo sobre bibliografía relacionada a la lengua y cultura Chipaya en Dedenbach-Salazar Sáenz (2007) y en bibliografía relativa al Pueblo Uru en Volveré, Revista Electrónica 29, 2008 http://www.iecta.cl/revistas/ volvere_29/bibliografia.htm 